Award Address

\title{
Fine Structure of Starch and its Relationship to the Organization of Starch Granules
}

\author{
Dexter French \\ Dept. of Biochemistry and Biophysics, \\ Iowa State University, Ames, Iowa, U.S. A.
}

A starch granule is a highly-organized body of size, shape, and chemical composition depending on the source. Variation is the rule. No two granules are identical, and very possibly no two branched starch molecules within a single granule are identical. Thus in dealing with starch we are confronted with a heterogeneity of populations for which it is tempting, though conceptually dangerous, to assign average properties-physical, chemical and even biological.

The present lecture is an attempt to present the author's viewpoint on the structure of starch and starch granules. This viewpoint cannot be regarded as being complete or final. Rather, it is intended to be a statement of some of the facts, some of the speculations, and some of the problems which must eventually be considered in arriving at a unitary picture of starch behavior. At the outset it is appropriate to acknowledge the excellent experimental work of the author's past and present co-workers, Dr. Abdullah, whose study on pullulanase and glycogen fine structure was essential to our understanding of the branching pattern of starch, Dr. Kainuma whose most excellent studies have provided the details of starch branching, and Dr. Watanabe who is currently working on the very difficult problem of the structure of Naegeli amylodextrin.

Technological applications of starch depend heavily on the colloidal character of aqueous starch or dextrin pastes, gels, or sols, or on the dried films made from such dispersions. It is a characteristic of starch, as well as of many other high polymers, that the properties of a given dispersion depend not only on the chemical constitution of the polymer, but also on its physical organization and prior treatment. Thus the common starch dispersions are in an unstable, or at most a metastable, state.

While the physical changes accompanying starch gelatinization, film formation and retrogradation are of great technological importance, the author will not explore these subjects further during this lecture. Likewise, the biosynthesis of starch granules is a subject of exceptional interest and importance, which will doubtless give us a much better understanding of starch granule organization. However, at the moment, our knowledge of starch biosynthesis permits us to see only in a general way how linear and branched molecules could be synthesized in solution. We do not understand how the linear amylose and branched amylopectin can be synthesized side-by-side, without the amylose becoming branched by the enzymes which branch amylopectin. Also, it is still an open question as regards the degree to which starch molecules are synthesized in solution, then deposited at the growing surface of the granule, or whether the granule surface itself represents the site of enzymic action, and the outer chains of the starch granule then comprise the "primers" for chain elongation. My colleague Dr. John F. Robyt has suggested that amylose may be biosynthesized by an "insertion" mechanism, i.e., one in which the amylose chain grows from the reducing end via an appropriate glycosyl "carrier". Such a synthetic pattern would result in amylose being laid down in the starch with the reducing group 


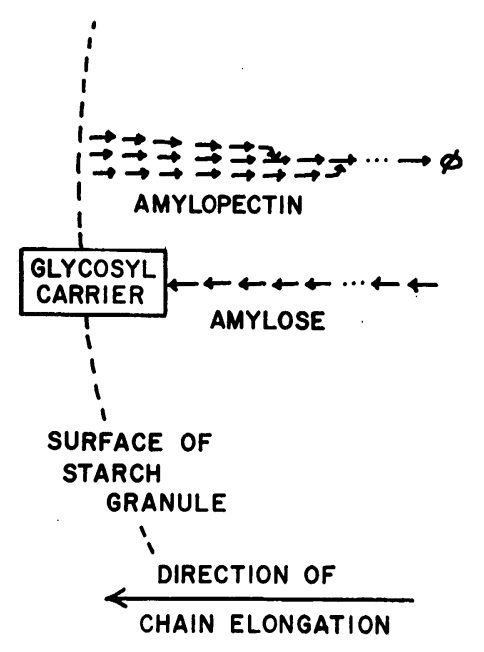

Fig. 1. Suggested mechanism for the side-byside synthesis of amylose and amylopectin at the growing surface of a starch granule. The amylopectin is synthesized by elongation at the non-reducing ends (" amylopectin synthetase”) followed by Q-enzyme. Amylose is synthesized through an insertion mechanism in which the amylose chain is glycosidically attached through its reducing group to an appropriate "carrier".

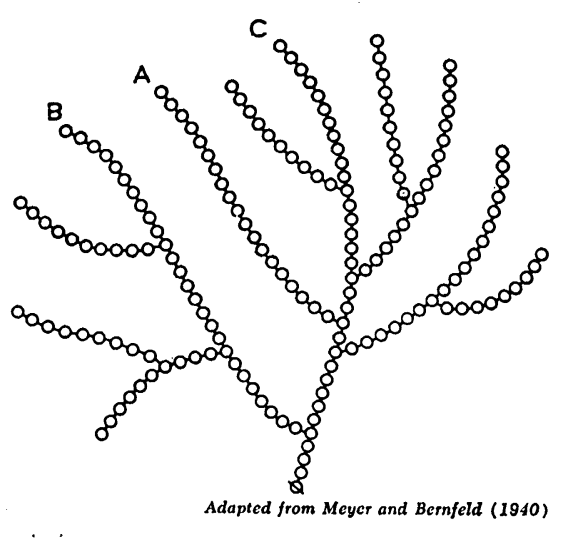

Fig. 2. Meyer "tree" formula for amylopectin (after Meyer and Bernfeld $\left.{ }^{2}\right)$ ).

toward the outside of the granule, whereas with amylopectin the reducing group is toward the inside of the granule (Fig. 1). Such an opposite polarity might mean that the amylose chains are protected from branching. This mechanism would not require a membrane or other compartmentalization to separate amylose synthesis from amylopectin synthesis. The enzymes involved in chain elongation would be of two substantially different types, possibly utilizing different donors (e.g. UDPG and ADPG). In principle either type of action could elongate either amylose or amylopectin. If the growing polysaccharide molecule were actually a part of the growing starch granule, chain elongation could only occur in a direction away from the granule surface. An insertion enzyme could only produce amylose, since the parts of the chain where branching reactions would have to occur would be "buried" underneath the granule surface. Conversely, for an enzyme with conventional chain elongation at the non-reducing end, the longer the chains become, the more they penetrate into the aqueous medium and become accessible to the action of branching enzyme. However, at the moment this suggestion is purely speculative for starch, although there are analogies in bacterial polysaccharide synthesis ${ }^{1}$.

Now it is appropriate to turn from these speculations to examine some of the fine details of starch structure.

\section{A. The Fine Structure of Starch}

The well-known fractionation procedure of Schoch separates starch primarily into two fractions : a more linear fraction ("A-fraction", amylose) and a branched fraction ("B-fraction ", amylopectin). Ideally, the linear amylose fraction should consist of molecules which are essentially identical in structure, varying only in chain length. The branched amylopectin 
molecules on the other hand, vary in molecular size, but more importantly they also vary in the details as regards the number of branches per molecule, the length of the branches, the distances between branches, and the arrangement of the branches into a pattern. Thus, for amylopectin, almost an infinite number of different molecular structures could be drawn. Clearly, it would be impossible to delineate the totality of structures. Even the problem of reconstructing the formula for a single molecule is presently hopeless. In the future, it may be possible to visualize the branching pattern of amylopectin through high resolution electron microscopy. However, at present, this is impossible. The best that can be done is to obtain statistical averages of the frequency of certain structural elements. In essence, this is the basis of the Meyer formula for amylopectin (Fig. 2) ${ }^{2}$. This is based upon (a) the end group value (average length of the unit chains); (b) the $\beta$ amylase limit; and (c) the "tier" concept, originally obtained by Meyer by serial degradation by $\beta$ amylase and a yeast "glucosidase". Such information gives average values for the lengths of the outer chains and the distances between branch points (lengths of inner chains). However, it fails to tell how much variation, if any, there is in numbers and lengths of the various elements.

Our experimental approach to this problem has been to degrade amylopectin (actually, waxy maize starch) by porcine pancreatic $\alpha$-amylase, isolate the branched limit dextrins, and determine their amounts and structures. It is well known that only the $\alpha-1,4-$ links in amylopectin are susceptible to action of $\alpha$-amylase. The $\alpha-1,6-$ links are not only totally resistant to $\alpha$-amylases, but they also confer resistance to some of the nearby $\alpha-1,4-$ links. This is illustrated by the action of $\alpha$-amylase on an isolated branch point in amylopectin $(\text { Fig. } 3)^{3}$.

If, during the initial stages of $\alpha$-amylase hydrolysis, one of the linkages $A$ is hydrolyzed, subsequent hydrolysis eventually leads to cleavage of linkage $B$, so that the limit dextrin always has a chain of two glucose units (resistant linkages $C$ ). On the distal (left hand) side of the main chain, structures which contain $D$ linkages will be hydrolyzed until either $E$ or $F$ is cleaved. The resistant structure contains either 0 or 1 glucose unit joined by a 1-4 linkage to the branch point. Similarly, labile linkages $C$ on the branch are cleaved until one of the linkages $H, I$ or $J$ is broken. Steric factors appear to require that $J$ cannot be broken unless $E$ or $F$ has previously been broken; moreover $\alpha$-amylase is unable to remove a single glucose unit from the non-reducing end of the molecule. These considerations account for the structures $\mathrm{B}_{4}-\mathrm{B}_{7}$ (Fig. 4).

Approximately $64 \%$ of the total number of branches in waxy maize starch are converted by $\alpha$-amylase to these singly-branched limit dextrins. The remainder $(36 \%)$ are converted

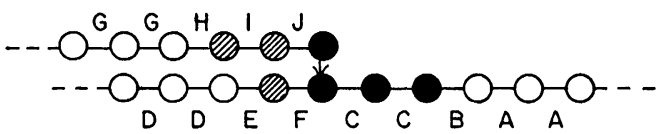

Fig. 3. Action of porcine pancreatic $\alpha$ amylase on an isolated branch point. The filled circles represent glucose units which remain in the limit dextrins; the shaded circles represent glucose units which are removed by amylase only with difficulty or not at all. The open circles represent easily-removed glucose units.

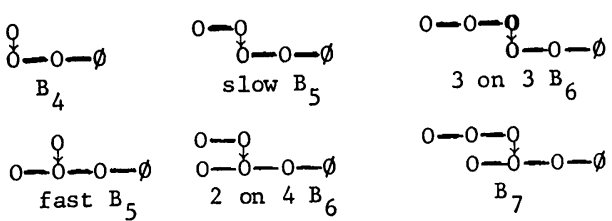

Fig. 4. Singly-branched oligosaccharides (limit dextrins) formed by action of porcine pancreatic $\alpha$ amylase on amylopectin or glycogen. The 3 on $3 \mathrm{~B}_{6}$ is slowly hydrolyzed to $\mathrm{G}_{2}+$ $B_{4}$, and $B_{7}$ is extremely slowly hydrolyzed to $\mathrm{G}_{2}+$ fast $\mathrm{B}_{5}$ by $\alpha$-amylase. 
into doubly-branched dextrins and still more complex dextrins.

The structures of the doubly-branched limit dextrins have been worked out by a combination of enzymic degradations, employing pullulanase, glucoamylase, $\alpha$-amylase and $\beta$-amylase in various combinations and sequences. The doubly-branched dextrins fall into two categories which we call the "Haworth" and "Staudinger" patterns (Fig. 5).

We presume that these branching configurations in the $\alpha$-amylase limit dextrins come from corresponding branching arrangements in the parent starch. Formation of such BB oligosaccharides indicates that starch itself contains branching configurations such as in Fig. 5.

Inspection of Fig. 3 indicates that Haworth and Staudinger structures such as in Fig. 6 would be cleaved by pancreatic amylase, and in fact no such compounds were isolated from the limit dextrins. However, we did obtain triply-branched oligosaccharides such as in Fig. 7.

No evidence was obtained for the more compact configurations as in Fig. 8. Although we could not state with certainty that such configurations are totally absent we feel that they are not numerically important to starch branching.

From these studies we conclude that a significant fraction of the inner chains of starch may be only one or two glucose units in length, far too short to be cleaved by $\alpha$-amylase. At the same time since the average inner chain length, according to Meyer, is about 8 glucose units, there must be some chains substantially longer than 8 glucose units, or as long as the average outer chain (12 glucose units).

\section{B. Conformations of Starch Chains}

It is rather ironical that the powerful method of X-ray crystallography has given us much information about the conformation of various artificial forms of starch (amylose complexes), but as of the present has told us little about the conformation of technologically important forms of starch in starch granules and retrograded starch. Ever since the pioneering work of Katz it has been known that starch granules and retrograded starch give

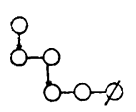

A

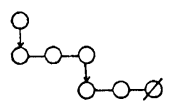

B

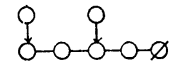

$\mathrm{C}$
Fig. 5. Doubly-branched configurations resistant to porcine pancreatic $\alpha$-amylase; $\mathrm{A}$ and $\mathrm{B}$ : Haworth configuration; C: Staudinger configuration.
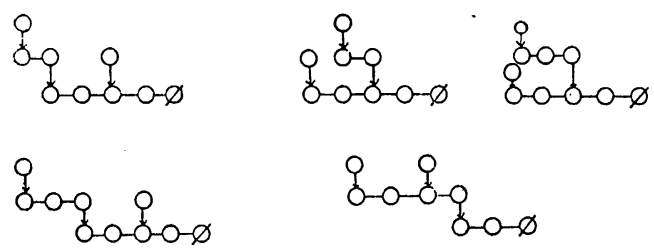

Fig. 7. Triply-branched oligosaccharides formed by action of porcine pancreatic $\alpha$-amylase on amylopectin.

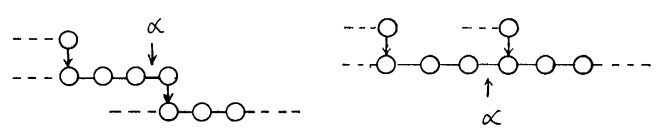

HAWORTH AND STAUDINGER CONFIGURATIONS WHICH ARE CLEAVED BY $\alpha$-AMYLASE

Fig. 6. Doubly-branched configurations susceptible to action of porcine pancreatic $\alpha$-amylase.

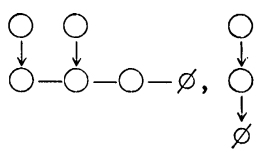

Fig. 8. More compact doubly-branched configurations; no evidence was obtained that these configurations occur in waxy maize starch. 
"A" and "B" type patterns $\mathrm{s}^{4}$. Yet, as of today, these patterns are not understood in spite of much work and the availability of excellent "fiber patterns" of both the A and B type. In 1939 Bear and French" declared that the data were "not very suggestive of spiral [compact helical] models for starch chains..." In 1943 Rundle, Daasch and French published an extended chain formula, which appeared reasonable at the time and was copied into many textbooks ${ }^{6}$. However, this formula required distorted bond lengths and bond angles, and cannot be accepted today. Similarly, practically all other proposals for the A-B structure fail to satisfy modern stereochemical requirements. The only exception is the hydrated helix of Blackwell, Sarko and Marchessault ${ }^{7}$. While this structure is stereochemically feasible, it requires an enormous amount of water of crystallization. We shall show that $\mathrm{A}-\mathrm{B}$ starch is essentially water-free, so the Blackwell structure also must be rejected.

Inasmuch as none of the proposed single stranded models agree with the $\mathrm{X}$-ray data, the author proposes consideration of double-stranded models, or twisted helices (Fig. 9). Spacefilling models of such helices are easily constructed with either "parallel" or "anti-parallel" chains. With parallel chains, the repeat period corresponds to approximately $10.5 \AA$ per three glucose units, in agreement with the $\mathrm{X}$-ray data (there are 6 glucose units per complete turn of each helix, corresponding to a period of $21 \AA$, but since the structure has a 2 -fold axis this period is halved giving $10.5 \AA$ ). Molecular models indicate a diameter of approximately $11 \AA$, too large to fit into the Rundle, Daasch and French unit cell but easily accommodated by the Schieltz ${ }^{8}$ unit cell. Moreover, the observed density of B starch $(1.6 \mathrm{~g} / \mathrm{cc})$ agrees with that calculated for the Schieltz cell with 12 glucose residues and no water per unit cell $(1.59 \mathrm{~g} / \mathrm{cc})$.

Unfortunately, the concept of double helices has not been rigorously tested, and at present it can only be regarded as a working hypothesis. However, the author is strongly attracted to the idea, and I know of no valid evidence against such a structure.

As regards anti-parallel double helices, models indicate that such structures from just as easily as the parallel arrangement. In fact, it is possible that a single molecule can fold back on itself to give a "hairpin" type of arrangement (Fig.10). If the molecule is folded back and forth many times it could initiate a type of crystallization consisting of anti-

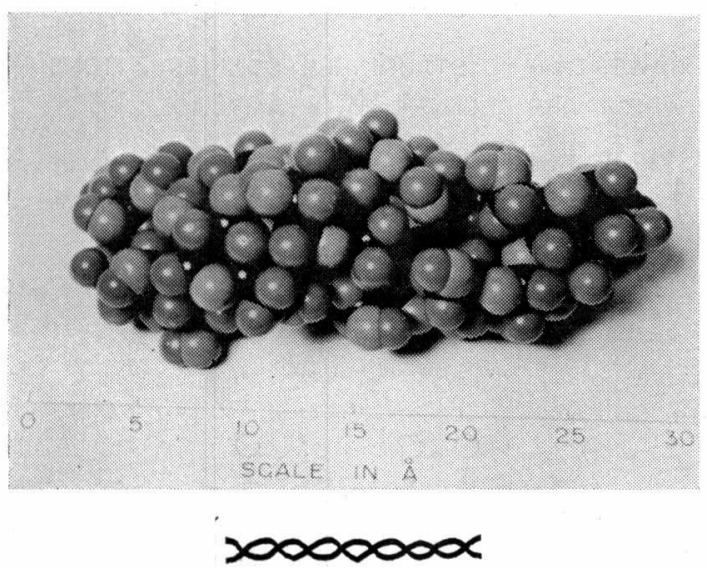

Fig. 9. Double helices: Above, space filling model; Below, symbolic representation.

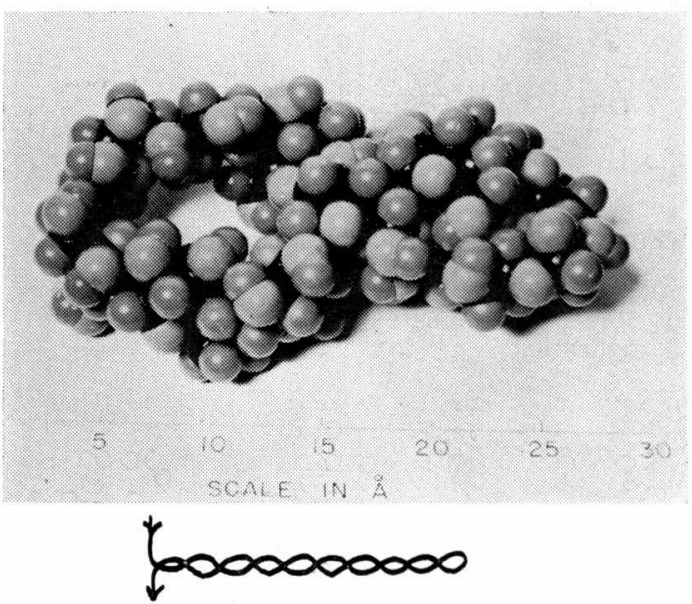

HAIRPIN ARRANGEMENT

Fig. 10. "Hairpin" model of double helix formed by a linear starch molecule folded back on itself. 


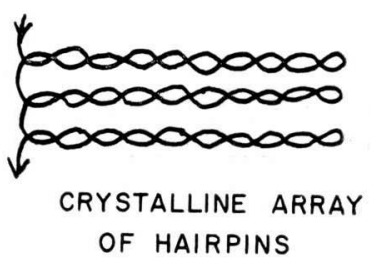

Fig. 11. One layer of a packing arrangement of folded amylose chain.
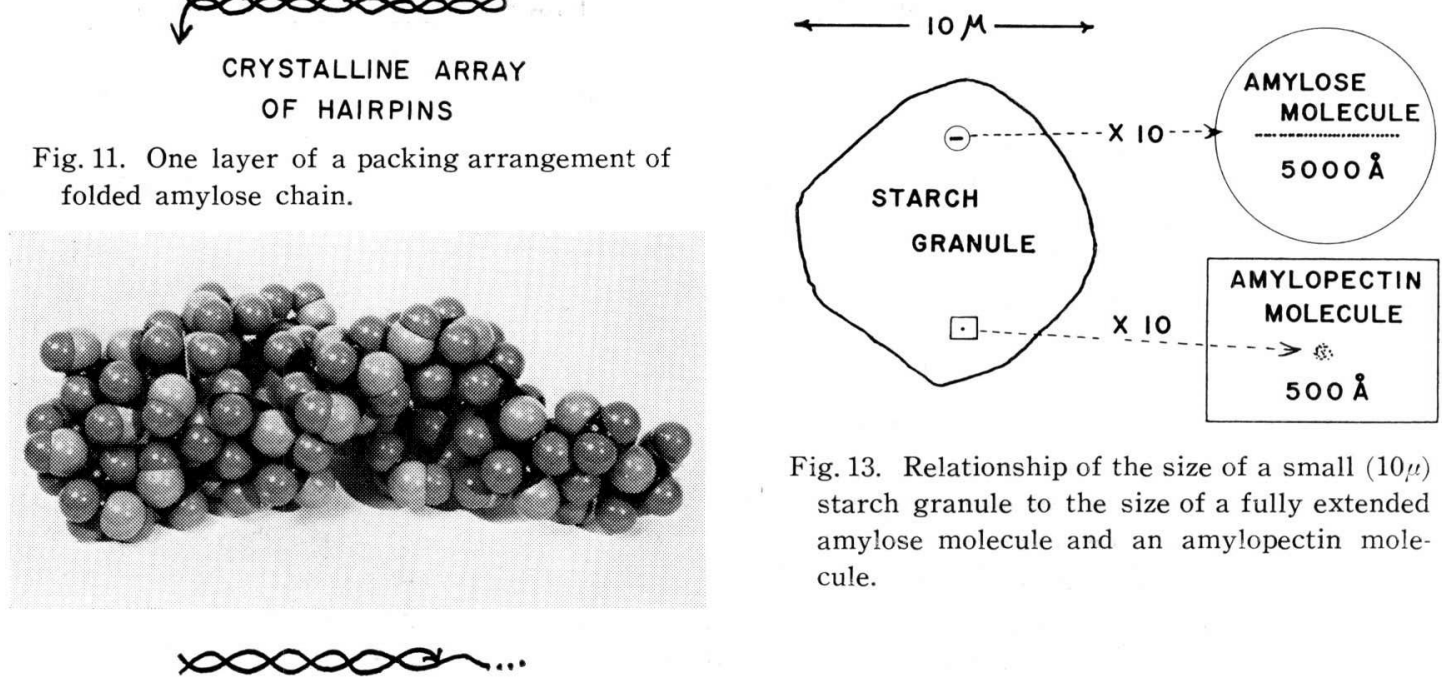

Fig. 13. Relationship of the size of a small $(10 \mu)$ starch granule to the size of a fully extended amylose molecule and an amylopectin molecule.

Fig. 12. Double helix originating from branch point. Above, space-filling model; Below, symbolic representation.

parallel chains packed together into a crystalline lattice as in Fig.11. It is well known that linear polymers have a high tendency to undergo chain folding ${ }^{9}$. Such an arrangement might well be consistent with the "tangling" of amylose chains in the retrogradation process.

Another concept which may be significant is that a branch point may well initiate double helix formation. Again, use of models indicates that two peripheral branches can originate from the same glucose unit, one at position 4 and the other at position 6 . These two chains can easily twist about each other to form a perfect double helix as in Fig. 12. It requires little imagination to picture more complex branching arrangements involving double, triple or higher branches, either alone or even with linear chains. Thus a wide range of double helical conformations is available.

\section{Dimensions of Starch Molecules}

In the sections which follow, we must keep in mind that starch granules are enormously large in comparison to starch molecules. If we examine a granule of corn starch for example, which is about 10 microns in diameter, it would have about $10^{11}$ glucose residues. If we assume that the average starch molecule contains about 1000 glucose units, this would permit $10^{9}$ starch molecules per starch granule. An amylose molecule of 1000 glucose units could be stretched out a slender chain $5000 \AA$, or $0.5 \mu$, in length. Of course, its conformation in solution would be much more compact. An amylopectin molecule, being branched, would be a few hundred $\AA$ in diameter. These relationships are indicated in Fig. 13.

It has been shown that the crystallite size in starch granules is of the order of $100-150$ $\AA^{10)}$. Thus it seems plausible that an individual molecule of amylopectin could comprise many crystallites. We shall return to this point when considering the relationship between 


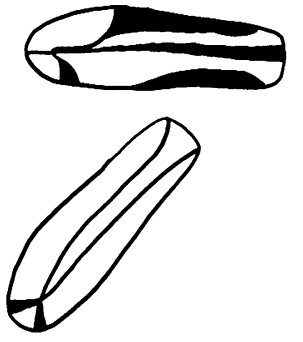

Polarization Pattern
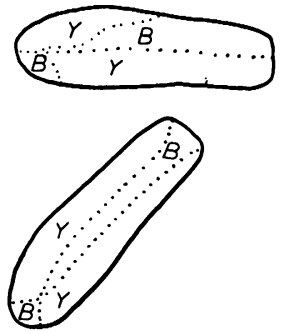

$1_{\text {st }}$ Order Red

Retardation

Patterns
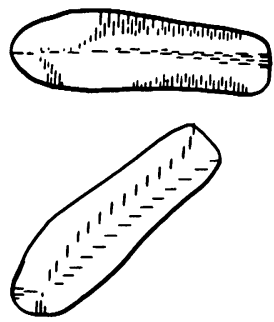

Molecular

Orientation

Fig. 14. Method of obtaining optical maps from birefringence of starch granules. At the position of the "dark cross" the average orientation of the starch chains is either parallel or perpendicular to the plane of the polarized light.

Naegeli amylodextrin and starch granule structre.

\section{Optical Maps of Starch Granules}

To elucidate the arrangement and orientation of starch molecular chains within starch granules, we have devised two techniques which depend on the optical properties of starch granules. The first is based on the birefringence of starch, a classical technique for obtaining information about the orientation of biopolymers. The method is based on two simple assumptions: (a) starch molecular chains are optically positive, that is, they have their highest index of refraction along the molecular axis, and (b) there is an averaging effect so that in any given element of volume of the starch granule there is a predominant orientation which in fact is a certain kind of weighted average of all the molecular chains in that volume element.

The fact that starch granules have a positive sign of birefringence indicates that the orientation of the molecules is in a preponderantly radial direction. More specifically, at the position of the "dark cross" the average orientation of the molecular chains is either parallel or perpendicular to the plane of polarization. The method for obtaining birefringence optical maps is illustrated in Fig. 14. To obtain optical maps, starch granules were photographed in a series of orientations, under the polarizing microscope. For each photograph, the molecular orientations were traced onto a single transparency. The combined tracings for all orientations of the starch granule constitute an "optical map" of the orientations of starch chains. Typical maps for various types of starch granules are shown in Figs. 15-17.

These maps confirm the general "radial" orientation of molecular chains, particularly in the least asymmetric or more spherical granules. However, the orientation may be more accurately described as being always perpendicular to the starch granule surface. This becomes particularly important for irregularly shaped granules, such as those of Dieffenbachia, which are often highly elongated or which may have large protuberances. Similarly, for the large disk shaped granules of wheat starch, the maps indicate clearly that the preponderant orientations are best described as being perpendicular to the surface. Incidentally, this accounts for the well-known fact that the large wheat starch granules show a very weak polarization cross, since in effect the ordinary direction of observation is along the 
Dieffenbachia Starch Granule
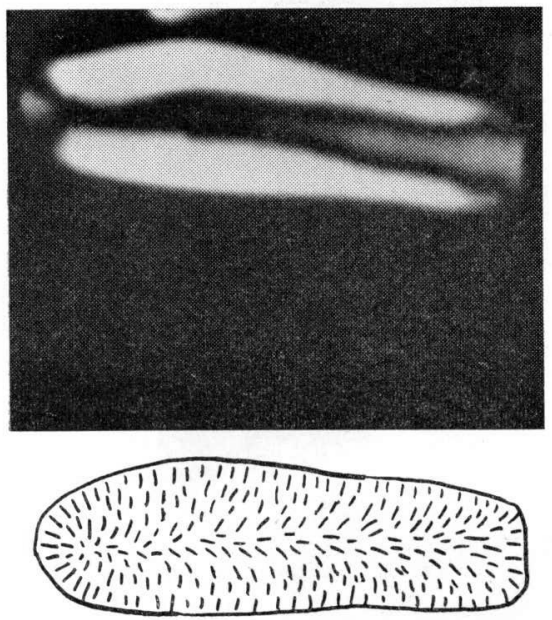

Above: Photograph, Crossed Polarizers Below: Orientation of Starch Chains

Fig. 15. Birefringence map of the orientation of starch chains of Dieffenbachia sp.. Above, view in light polarized at $45^{\circ}$ to horizontal; below, optical map.

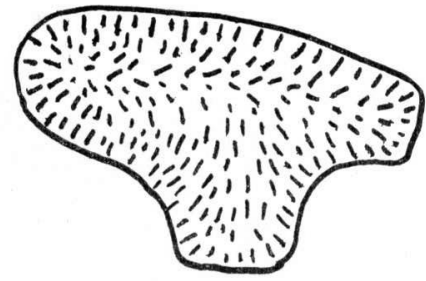

Fig. 16. Birefringence map of the orientation of starch chains in a Dieffenbachia starch granule with protuberance.

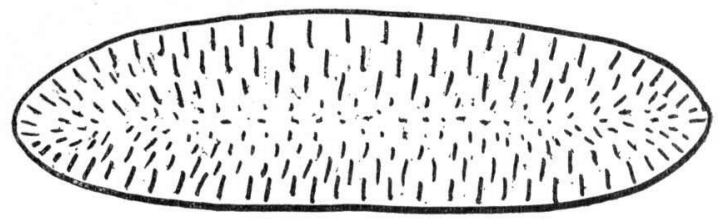

Fig. 17. Birefringence map of the orientation of starch chains in a wheat starch granule; view in "thin" direction.

Dichroism of Iodine Complexes
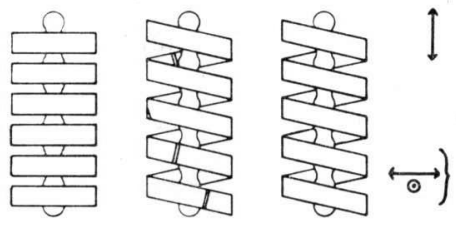

INTENSE

ABSORPTION

Fig. 18. Scheme showing the relationship between orientation of the iodine chain in carbohydrate iodine complexes and the absorption of polarized light (linear dichroism).

molecular axis. However, when such granules are turned on edge, they become brilliantly birefringent.

The second type of optical map is based on the strong linear dichroism of the oriented amylose-iodine complex. The fundamental concept is that the amylose-iodine complex has a very high absorbance for polarized light in which the electric vector is parallel to the axis of the amylose-iodine helical complex (Fig. 18). For light having the electric vector perpendicular to the amylose-iodine helix axis, there is little or no absorption in the visible range of wavelengths. By using plane polarized light, therefore, a starch preparation stained with iodine absorbs primarily only where the molecular chains lie in the direction of the plane of polarization. The application to maltohexaose-iodine complex is illustrated in Figs. 19-23.

For iodine-stained native starch granules, the optical absorption is so intense that the granules are opaque. If one reduces the intensity of the iodine stain, it appears that only a surface layer becomes stained, and it shows little if any dichroism. Similarly, if iodine 


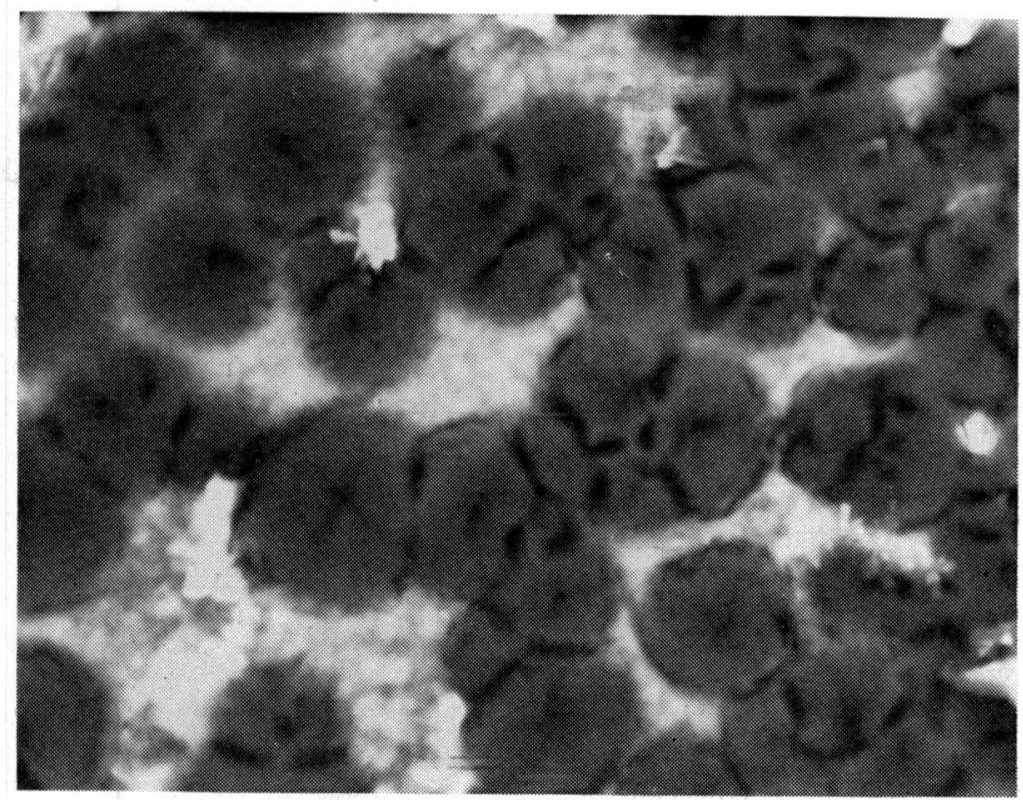

Fig. 19. Maltohexaose-iodine complex, disco-crystals, unpolarized light.

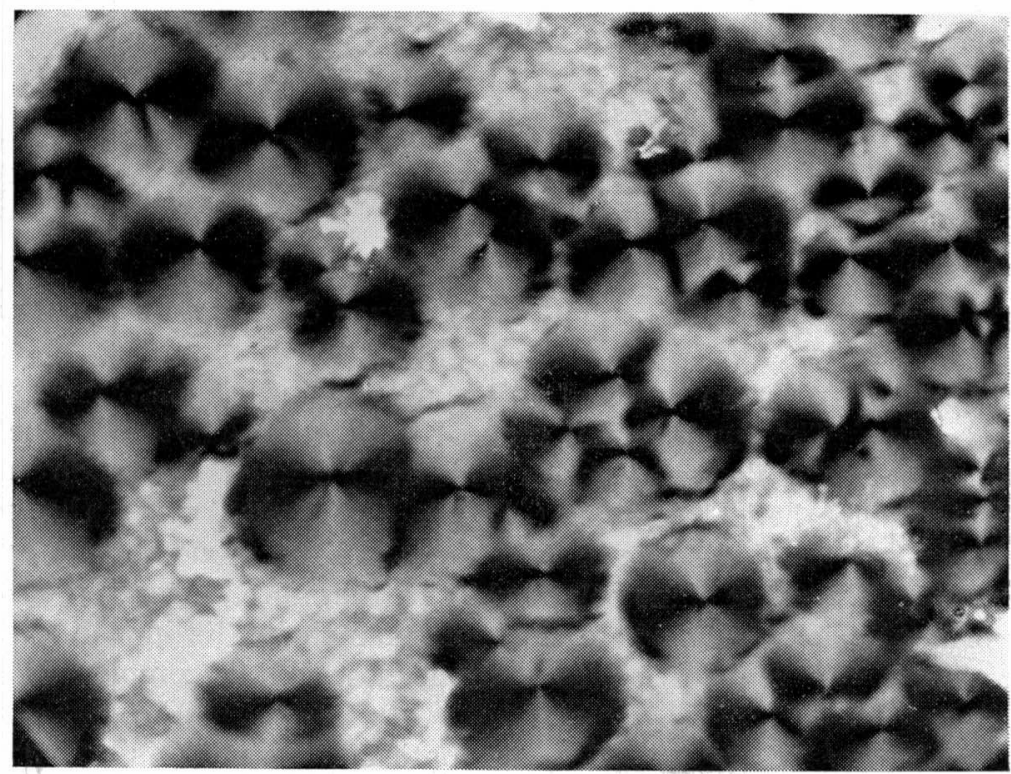

Fig. 20. Maltohexaose-iodine complex, the same field as in Fig. 19 , plane (horizontal) polarized light. 


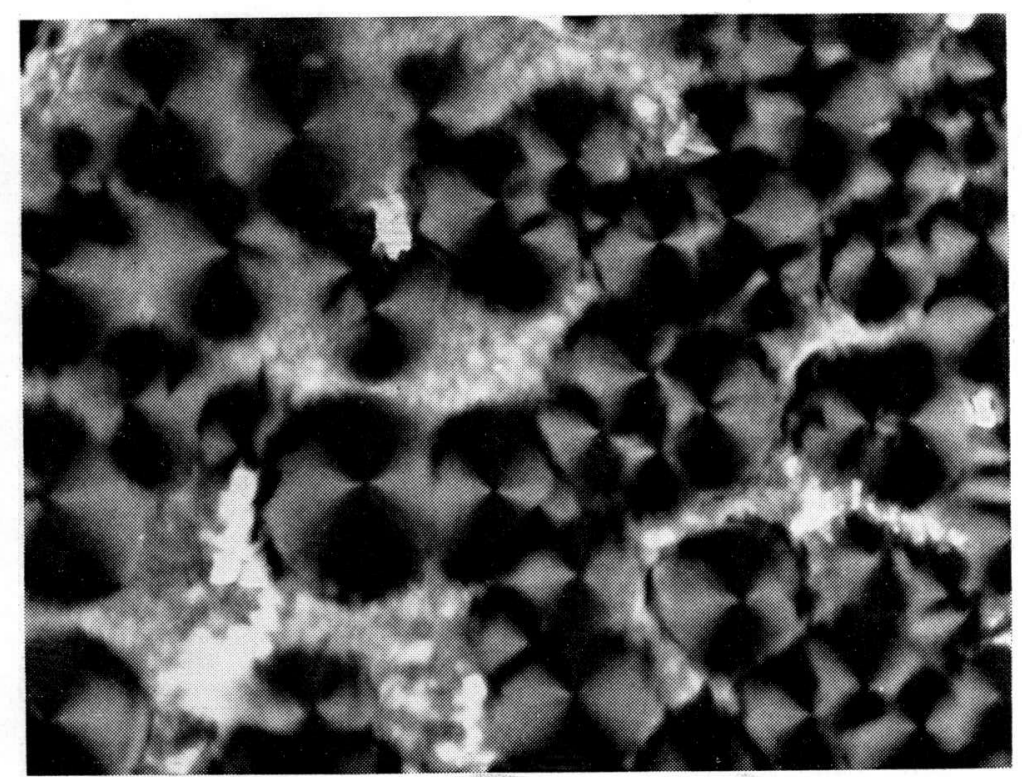

Fig. 21. Maltohexaose-iodine complex, the same field as in Figs. 19 and 20, plane (vertical) polarized light.

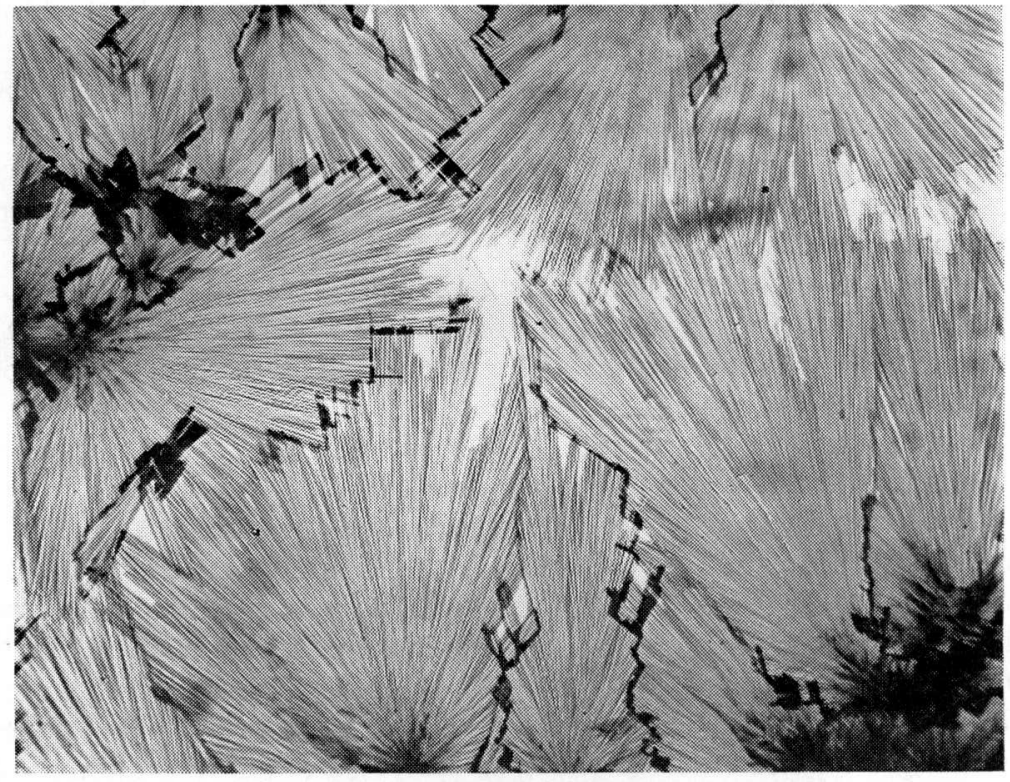

Fig. 22. Maltohexaose-iodine complex, crystals, unpolarized light. 


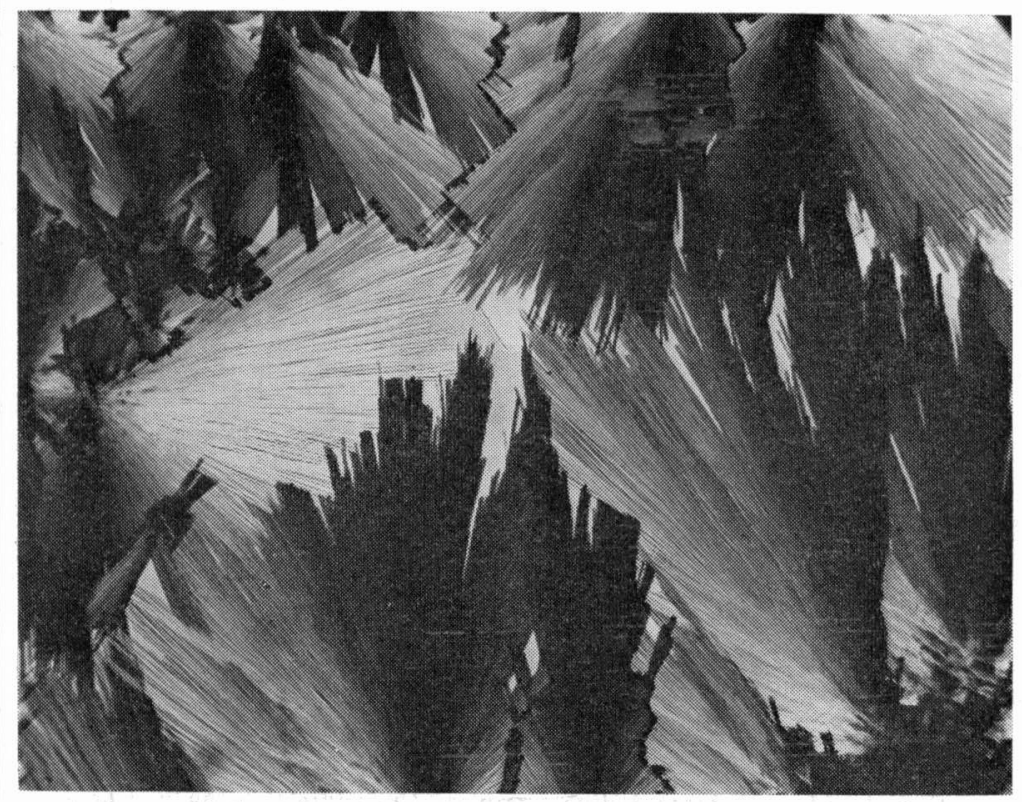

Fig. 23. Maltohexaose-iodine complex, the same? field as in Fig. 22, plane (vertical) polarized light.

is slowly removed from heavily-stained granules, they usually show little or no dichroism.

However, Prof. Darryll E. Outka suggested to the author that it might be possible to cut thin sections of starch granules (as for electron microscopy) and stain the resulting sections. Accordingly we cut serial sections from starch granules embedded in an epoxy resin, using a diamond knife, and stained the sections using dilute iodine-iodide solution. Photographs of some of the sections are shown in Fig. 24. We were particularly fortunate to obtain serial sections from several single starch granules.

Dichroism of the stained sections is most intense with the central section. While the dichroism is not as intense or as dramatic as the birefringence of whole starch granules, the dichroism indicates a general orientation of the amylose-iodine complex in a radial fashion. Dichroism decreases with sections farther removed from the center, and finally the sections nearest the surface show no significant dichroism whatsoever.

Dichroism optical maps of sections were prepared in a manner similar to that used for the birefringence maps (Fig. 25). As with birefringence, dichroism indicates a preponderant alignment of the amylose chains in a direction perpendicular to the surface of the granule. For such an orientation it is to be expected that with tangential sections, the direction of viewing will be along the direction of the amylose-iodine helix, hence the absorption will be weak (as observed) and not oriented in any particular direction.

The low degree of orientation of amylose is somewhat puzzling, in view of the strong birefringence of starch granules. Is it possible that the iodine stains primarily the amylose of the non-crystalline, amorphous or gel regions of the starch granule? In contrast to the crystalline regions, it is possible that the net orientation of starch chains in the gel region is very imperfect, and hence the iodine complex is poorly oriented. The amylose may be well-oriented in the crystallites. Here, however it reacts poorly with iodine so that the crystallite amylose contributes little to the total amylose-iodine complex. 


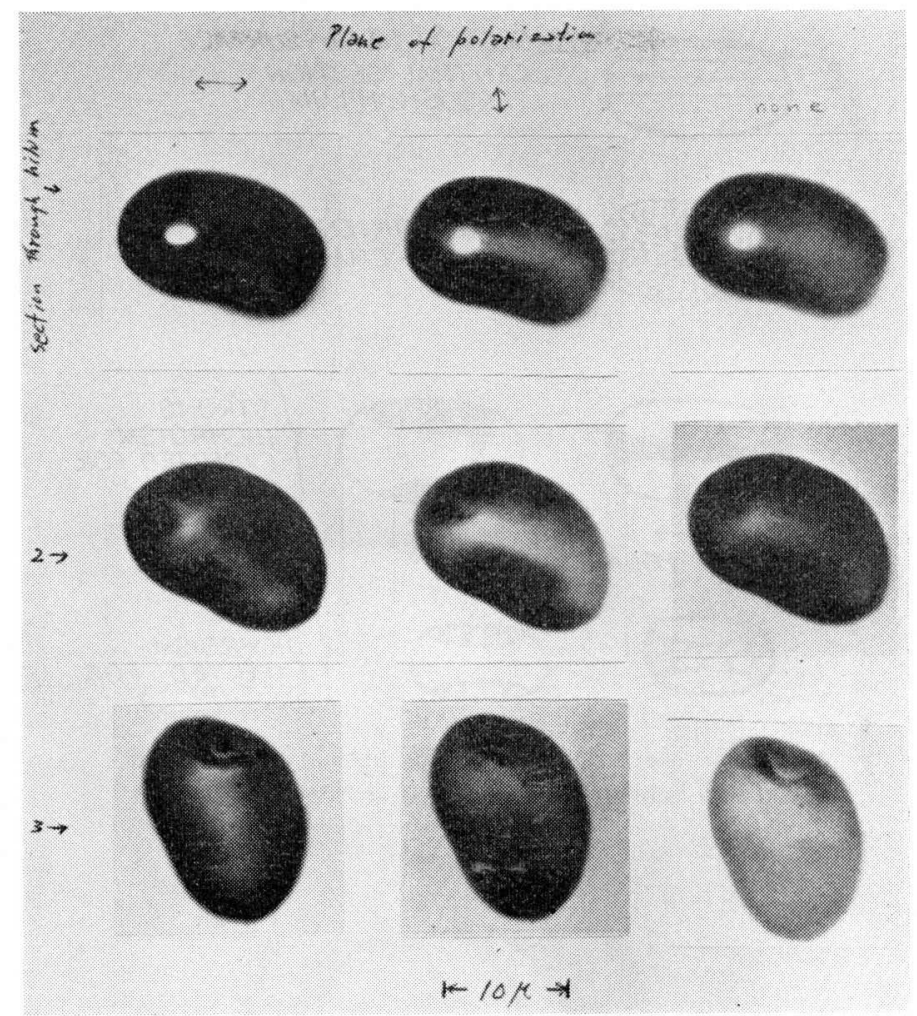

Fig. 24. Iodine-stained thin sections of potato starch granule. Top: section through hilum (central section); middle: adjacent section; bottom: section closer to surface; left, plane of polarization horizontal $\leftrightarrow$; center, plane of polarization vertical $\uparrow$; right, unpolarized light.

\section{E. Naegeli Amylodextrin and its Relationship to Starch Granule Structure}

Native Naegeli amylodextrin is the insoluble residue of starch which remains after protracted treatment of starch granules with $15 \% \mathrm{H}_{2} \mathrm{SO}_{4}$ at room temperature. It represents a certain type of starch modification of which "Lintner soluble starch" is the first stage. After a few days of acidic treatment, starch will no longer form a paste, but dissolves to give a more or less clear, non-viscous solution. After 3 months of treatment, the rate of erosion of the starch has greatly decreased, and we have arbitrarily adopted 3 months as the standard in our preparations. However, the acid may be left in contact with the granules for at least 6 years without the residual starch being dissolved. Thus, whatever is the structure of the Naegeli amylodextrin, it is certainly exceedingly resistant to hydrolysis by aqueous acid in the cold.

Under the microscope, the Naegeli amylodextrin retains much of the appearance of the original starch. It is highly birefringent and shows the same type of retardation pattern (using the first order red plate) as does starch. Potato starch in particular retains its granular appearance, while corn starch has more of a tendency to disintegrate into fine insoluble particles.

As previously mentioned, after a few days of acidic treatment the residual starch dissolves readily in hot water. After 3 months of treatment, solutions of Naegeli amylodextrin are 

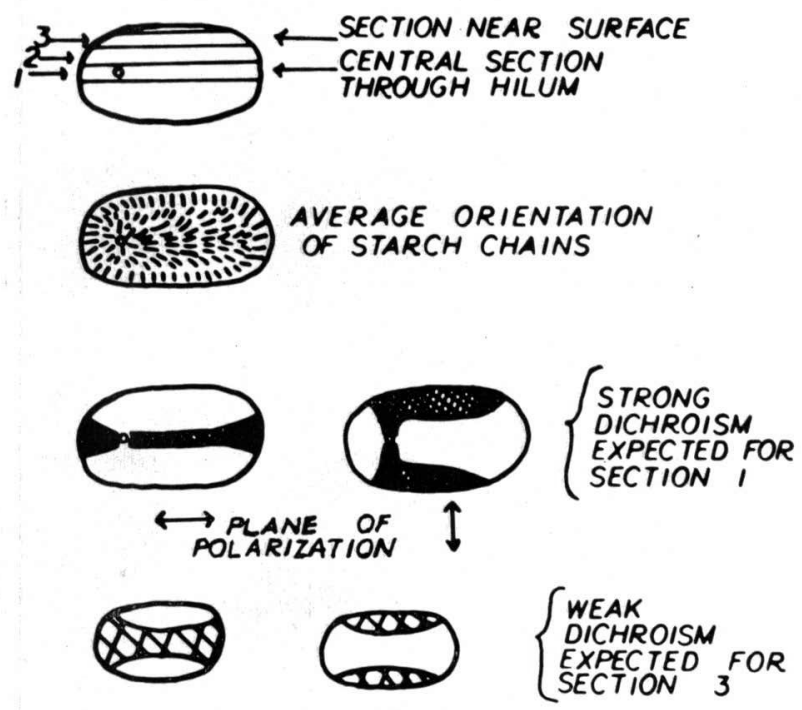

Fig. 25. Method of obtaining optical maps from dichroism of iodine-stained starch granule sections.

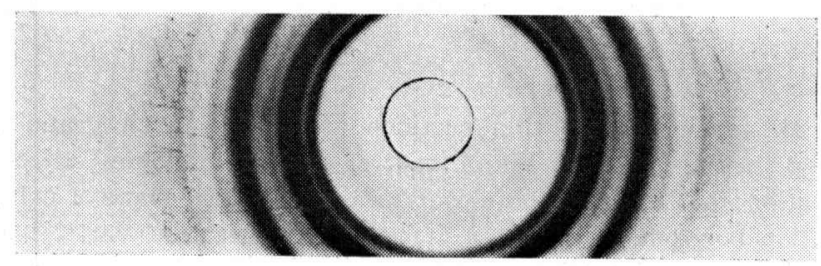

Fig. 26. X-ray diffraction A-pattern of Naegeli amylodextrin crystallized from $60 \%$ methanol.

colored red-violet with iodine (depending on the amount of iodine added). However, the undissolved native amylodextrin stains only a light yellow-brown. The $\mathrm{X}$-ray diffraction pattern is similar to that of the original starch, except that it is usually sharper (Fig. 26). Solutions of amylodextrin can be readily "crystallized" by adding methanol, or by merely concentrating or freezing an aqueous solution.

The average molecular size, as determined by reducing value, is about 25 glucose units. The solutions are degraded to about $75-80 \%$ maltose by $\beta$-amylase. Fractionation by Sephadex gel filtration gives 3 broad, poorly separated fractions (Fig. 27). Paper chromatography indicates that there are some molecules as small as 7 or 8 glucose units; recrystallization incorporates even such small molecules into the crystalline structure.

Initially Dr. Kainuma, and now Dr. Watanabe, have been making a very determined effort to learn the structure of this heterogeneous material. It is our working hypothesis that Sephadex Fraction I (highest molecular weight) represents the most complexly-branched configuration, for example, doubly-branched, triply-branched, and higher structures. The intermediate Fraction II has an average molecular size of about $20-30$ glucose units and represents primarily singly-branched arrangements. Fraction III, the lowest molecular 


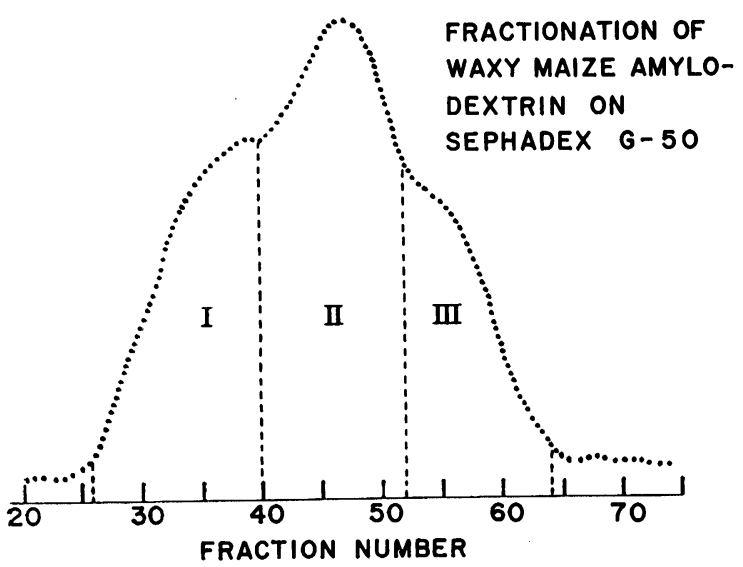

Fig. 27. Elution diagram of waxy maize amylodextrin on Sephadex G-50. I, highest molecular weight, complexly-branched; II, DP 20-30, singly-branched; III, DP 10-20, linear.

\author{
SUGGESTED STRUCTURES FOR \\ SEPHADEX G-5O FRACTIONS FROM \\ WAXY MAIZE AMYLODEXTRIN
}

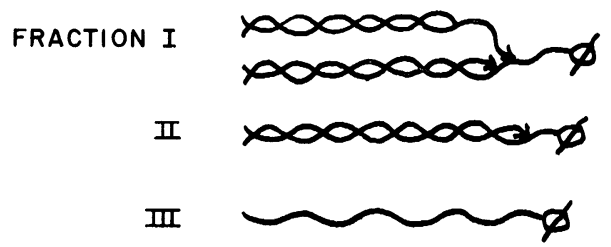

Fig. 28. Suggested structures for Sephadex G50 fractions from waxy maize amylodextrin.

weight component, contains most of the linear molecules including some oligosaccharides of less than 10 glucose units.

The above working hypothesis can be depicted as in Fig. 28. Up till now we have not been able to obtain pure fractions of linear or singly-branched dextrins. However, much of our experimental work is in harmony with this hypothesis. We conclude that the native amylodextrin represents the crystalline regions in starch granules which are exceedingly resistant to acid hydrolysis in the cold. Surprisingly, the amylodextrin retains a significant amount of branching, although we have not as yet measured the actual degree of branching. The $\alpha$-amylase limit dextrins contain doubly-branched oligosaccharides similar to those obtained from native starch. The branch points themselves, though constituting structural irregularities and hence incapable of crystallization in the conventional sense, are nonetheless linked to the truly crystalline part of amylodextrin through linkages which are exceptionally resistant to acidic hydrolysis in the cold. It is the author's opinion that these observations are consistent with a double helical structure, originating in some instances from a branch point. However, the absolute determination of the polysaccharide conformation must ultimately depend on one of the powerful physical methods of structure analysis such as X-ray diffraction.

\section{F. Proposed Models for Amylopectin}

One of the earliest models for starch granules is the trichitic model proposed in 1895 by A. Meyer (Fig. 29). ${ }^{11)}$ For starch, no model is possible without knowing something of the composition and chemical structure of the starch molecule. In 1895, the concept of high polymers had not been developed, and Meyer could not have considered the relationship of his structure to complex macromolecules such as amylopectin. Since that time, many formulas have been proposed, such as the Haworth "laminated" formula, ${ }^{12}$ and the Staudinger "comb" or "herringbone" formula. ${ }^{13)}$ Finally the celebrated K. H. Meyer "tree" formula (Fig. $2)^{2)}$ has been very important in focusing attention on the details of amylopectin structure, and has been very successful in accounting for many aspects of amylopectin behavior. However, it is again appropriate to consider various geometries for starch, bearing in mind 


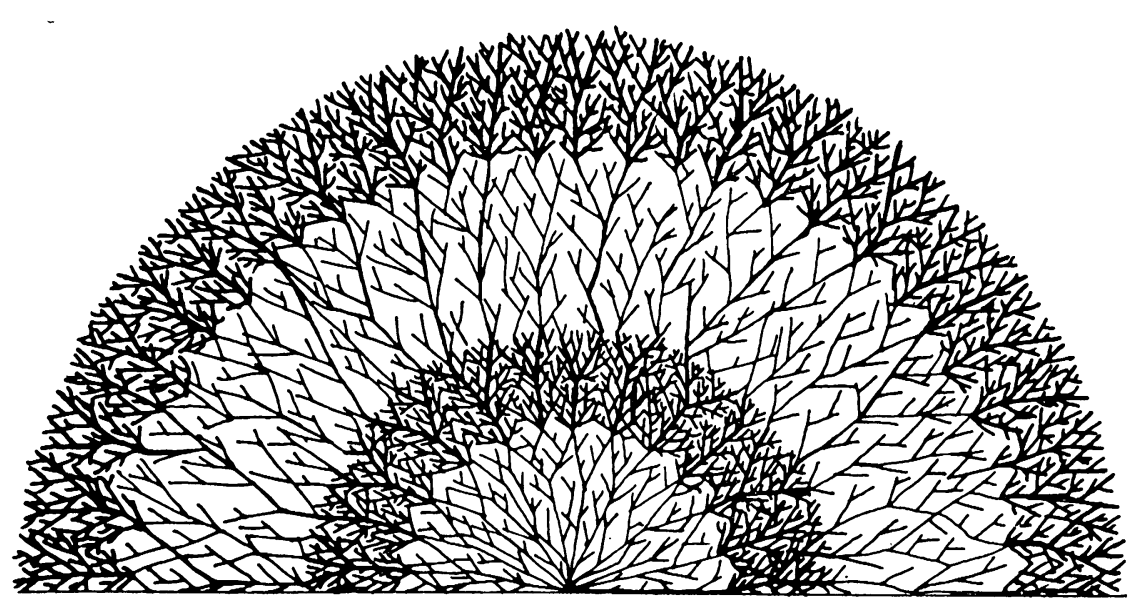

Fig. 29. Trichitic model of starch granule proposed by A. Meyer in 1895.

that any acceptable picture must now account for a much wider set of experimental facts.

There are several basic defects of the Meyer tree formula. For example, at least as usually depicted, the Meyer model assumes a more or less regularly re-branched structure, with inner chains of about 8 glucose units. Such a model becomes space-limited after a few "tiers" of re-branching and would then be a dense spherical molecule. Viscosity measurements on amylopectin indicate, on the contrary, that it is either highly asymmetric, or highly hydrated, or both. ${ }^{14}$ ) The maximum size calculated for such a regularly rebranched structure assuming a normal degree of hydration, would be about $700 \AA$ in diameter, with a molecular weight in the range of $30-80 \times 10^{6}$. Probably this size would accommodate all but the largest amylopectin molecules. After action of $\beta$-amylase, the limit dextrin of amylopectin is still highly asymmetric. This indicates that the limit dextrin and therefore the parent amylopectin also, are constructed on an asymmetric framework rather than a regularly re-branched structure.

The production of doubly-branched limit dextrins by $\alpha$-amylase hydrolysis of amylopectin would be impossible if these were a more or less uniform inner branch length. Even if some of the inner branches were as short as 3 or 4 glucose units, this would still permit total hydrolysis by $\alpha$-amylase to produce singly-branched oligosaccharides. As pointed out previously, since some of the inner chains are as short as 1 or 2 glucose units, then at least an equivalent number must certainly be longer than the average. Two types of amylopectin structures would accommodate the above situations. The first is a modified trichitic structure, similar to the Meyer structure except without a regular rebranching pattern (Fig. 30).

Such a structure could expand indefinitely from the reducing end of the molecule, with branching occurring as space permits. Lengths of branches would be regulated in part by enzyme specificity, but also by the amount of space available. Availability of the sugar precursor for polysaccharide (e. g. ADPG, UDPG, G-1-P, etc.) would also be important in determining the relative effective reactivity of enzymes involved in chain elongation, chain transfer (branching) and chain shortening. Possibly diurual variations in temperature, availability of precursors and energy sources such as ATP also play a role in establishing the final pattern of the amylopectin molecule.

Another molecular pattern which deserves serious consideration is the "cluster" (racemose) 


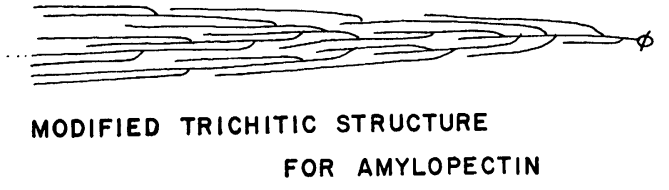

Fig. 30. Modified trichitic model for amylopectin molecule.

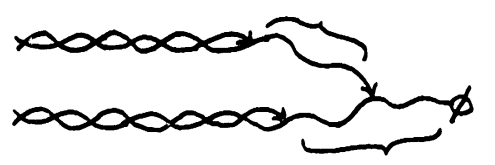

NON-RESISTANT AREAS IN DOUBLE HELICAL BRANCHED STRUCTURE

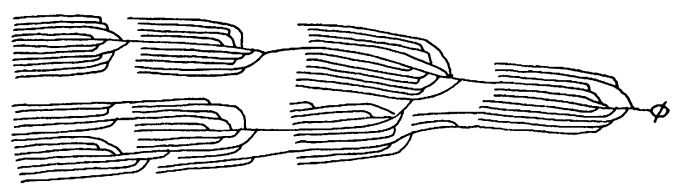

CLUSTER (RACEMOSE) HYPOTHESIS OF AMYLOPECTIN STRUCTURE

Fig. 31. Cluster (racemose) model for amylopectin molecule.

Fig. 32. Non-resistant areas in double helical branched structures.

pattern (Fig.31). Such a pattern could originate if parts of the amylopectin molecule crystallize during growth, thus sterically blocking off that portion from further chain elongation. Such a molecular pattern would have alternating crystalline and amorphous regions, the dimensions of the crystalline domain being of the order of the average outer chain length of amylopectin ( $c a .12$ glucose units or $50 \AA$ ). Such a cluster pattern would be in harmony with the formation of soluble starch and Naegeli amylodextrin. According to this concept, the initial acidic attack on starch granules would be on the relatively unprotected crystalline regions. Such an attack would lead to rapid breakdown in molecular size with the formation of amylopectin "sub-units", rather than a random fracture process. Incidentally, the subunits would be of dimensions similar to those found for starch crystallites. Further erosion of the structure will lead to cleavage of the least-protected interbranch regions-those which are incapable of forming double helices, for example (Fig. 32).

The biochemistry of the initiation of starch molecules, either amylose or amylopectin, is obscure. Chloroplasts, and presumably amyloplasts as well, have an abundant supply of linear oligosaccharides in the range of $2-10$ glucose units and higher. While the function of these is unknown, they could serve as "primers" for the initiation of starch molecules. Alternatively, individual macromolecules could originate from the cleavage of larger molecules and thereby become initiators for further molecular growth.

It has been seriously suggested that amylopectin molecules do not appear as such during starch biosynthesis, but represent either natural degradation products or artifacts of preparation. Such degradation might occur for example if there were weak or strained glycosidic bonds which readily undergo cleavage.

\section{G. The Gel Phase of Starch Granules}

Estimates of the "crystallinity" of starch granules vary, but all authors agree that starch is only partly crystalline. ${ }^{15}$ ) It is the author's viewpoint that the starch granule may be considered to consist of crystalline regions embedded in a gel matrix. The crystalline regions give rise to the $\mathrm{X}$-ray pattern and optical birefringence, and are relatively resistant to chemical and biochemical attack. These crystallites do not swell significantly during hydration of starch granules at temperatures below the gelatinization temperature. By contrast, the gel region is less dense, more open, much more susceptible to chemical and enzymic attack, absorbs water readily either from water vapor or from liquid water, and swells very substantially, the increase in granule volume being more or less in proportion 
to the amount of water taken up.

Penetrability studies of starch granules indicate that many small molecules (e. g. salts, amino acids) are readily absorbed by native starch granules whereas complex dyes (e. g. congo red) cannot be taken up unless the starch is swollen or damaged. Fatty acids which are incorporated into the cereal starch granules during the biosynthesis cannot be removed from unswollen granules by the usual lipid solvents, presumably because the dry gel structure is too dense and compact to admit the organic solvent or allow trapped and complexed fatty acid to escape. However, after the gel structure has been swollen (by water or other hydrophilic solvent) the structure is opened sufficiently that the fatty acid can be slowly extracted.

During the biosynthesis of starch granules they are in an aqueous medium, and the gel phase is in a maximum state of hydration. During drying, the gel phase contracts very strongly, and sets up heavy strains within the granule. These strains often rupture the starch granule, producing cracks and fissures. These strains are even sufficient to break covalent bonds so that strongly dried starch has been degraded from its native state. Severe drying weakens or destroys the X-ray diffraction pattern of some starches.

Although various nucleotides have been extracted from starch, no definitive studies have examined whether G-1-P, UDPG or ADPG can actually penetrate beyond the surface layer of the starch granule. This question relates strongly to the mechanism of the biosynthesis of starch. If growth of starch granules were by intussusception, this would require the permeation of starch precursors through the gel matrix. It has been reported that starch synthetase is firmly attached to starch granules and may be located in part in the interior of the granule. If so, it would be an inclusion in the gel phase, and to be reactive it would require that the substrates would diffuse through the gel phase. Incidentally, if polysaccharide is actually synthesized within the interior of a starch granule, one would expect that it would be poorly oriented. As indicated previously, the amylose of starch granules is not nearly as highly oriented as are the more crystalline regions.

Whatever be the exact nature of the gel phase of starch granule, it is not as compact as the crystallite region. If the crystalline regions are regarded as having a density of about 1.6, the amorphous gel regions have a density of about 1.45 so that the average density for the entire starch granule is about 1.53 .

\section{H. Conclusion}

In the preceding, I have attempted to present my ideas of some facets of starch structure and how they relate to the organization of starch granules. Hopefully at some future time it will be possible to test the more speculative suggestions, and to obtain more accurate information regarding structural details of starch. Some of the concepts discussed are on a firm experimental basis-for example, the fine structure of amylopectin branching leading to doubly and triply branched oligosaccharides, the crystalline character of amylopectin in starch granules, leading to formation of crystalline Naegeli amylodextrins, and the gel phase which is initially responsible for water uptake and swelling. Other concepts such as the double helical twisting of starch chains, the "cluster" theory of amylopectin structure and the insertion mechanism for amylose biosynthesis are more speculative, and these concepts need much experimental testing. Hopefully some of you will be able to test these ideas, and if necessary correct them or improve on them, so that we may all have a better understanding of starch structure and behavior.

In closing, let me express to you my appreciation for your kindness to me, and for allowing me to present these ideas to you. I hope that we can build more bridges between the nations of the world. The opportunity for scientists of different nations to meet in 
friendship and share ideas is one of the strongest bridges I can imagine, and I am grateful to you for giving me such an opportunity. I thank you.

\section{REFERENCES}

1) K. H. Ebert and G. Schenk, Advances in Enzymology, 30, 179 (1968).

2) K. H. Meyer and P. Bernfeld, Helv. chim. Acta, 23, 875 (1940).

3) D. French, Bull. Soc. Chim. biol., 42, 1677 (1960).

4) J. R. KATZ and J. C. Derksen, Z. physik. Chem., A165, 228 (1933).

5) R.S. BeAR and D. French, J. Am. Chem. Soc., 63, 2298 (1941).

6) R. E. Rundle, L. DaAsch and D. French, J. Am. Chem. Soc., 66, 130 (1944).

7) J. Blackwell, A. Sarko and R. H. Marchessault, J. Mol. Biol., 42, 379 (1969).

8) N. C. Schieltz, Quat. Colo. School Mines, 60, (40) 84 (1965).

9) P. J. Flory, J. Am. Chem. Soc., 84, 2857 (1962).

10) S. HizukURI and Z. NikUni, Nature, 180, 436 (1957).

11) A. MEYER, Untersuchungen über die Stärkekörner, Jena, Gustav Fischer, 1895.

12) W. N. Haworth, E. L. Hirst and F. A. Isherwood, J. Chem. Soc., 1937, 577.

13) H. Staudinger and E. Husemann, Ann., 527, 195 (1937).

14) C. T. Greenwood, Advances in Carbohydrate Chem., 11, 335 (1956).

15) C. Sterling, The Structure of the Starch Grain, Chapter 4 in Starch and its Derivatives, 4 th Ed., J. A. Radley, Ed., Chapman and Hall Ltd., London, 1968. 